BULLETIN (New Series) OF THE

AMERICAN MATHEMATICAL SOCIETY

Volume 43, Number 2, Pages 139-161

S 0273-0979(06)01081-0

Article electronically published on February 8, 2006

\title{
THE NOTION OF DIMENSION IN GEOMETRY AND ALGEBRA
}

\author{
YURI I. MANIN
}

\begin{abstract}
This talk reviews some mathematical and physical ideas related to the notion of dimension. After a brief historical introduction, various modern constructions from fractal geometry, noncommutative geometry, and theoretical physics are invoked and compared.

Glenn Gould disapproved of his own recording of Goldberg variations.

"There is a lot of piano playing going on there, and I mean that as the most disparaging comment possible." NYRB, Oct. 7, 2004, p. 10
\end{abstract}

\section{$\S 0$. INTRODUCTION}

0.1. Some history. The notion of dimension belongs to the most fundamental mathematical ideas. In Western civilization and our school system, we become exposed in early life to the assertion that the dimension of our physical space is three (and somewhat later, that time furnishes the fourth dimension).

However, what does such a statement actually mean?

The mental effort needed to grasp the meaning of "three" in this context is qualitatively different from the one involved in making sense of a sentence like "There are three chairs in this room." Counting dimensions, we are definitely not counting "things".

Even if we make a great leap to abstraction and accept Cantor's sophisticated definition of a whole number as a cardinality of a finite set, life does not get much easier. A Cantorian set is supposed to be "any collection of definite, distinct objects of our perception or our thought." But what exactly are these "distinct objects", these "non-things" which we take from our physical space and project into our mind?

Euclid (ca. $300 \mathrm{BC}$ ), as some great thinkers before and after him, taught us not to bother so much about what things "are" but rather how to think about them orderly and creatively.

Let us reread his often quoted passages where dimension is indirectly involved. I use the delightfully archaic rendering of [He]:

From BOOK I, On Plane Geometry:

1. A point is that which has no part.

Received by the editors April 24, 2005.

2000 Mathematics Subject Classification. Primary 14H10, 14N10.

Based on the talks delivered at the AMS sectional meeting, Northwestern University, October 2004; and Blyth Lectures, University of Toronto, November 2004.

(C)2006 Yuri I. Manin 
2. A line is breadthless length.

3. The extremities of a line are points.

$[\ldots]$

5. A surface is that which has length and breadth only.

6. The extremities of a surface are lines.

From BOOK XI, On Spatial Geometry:

1. A solid is that which has length, breadth, and depth.

2. An extremity of a solid is a surface.

Two observations are immediate. First, Euclid directs our imagination to "kitchen physics": e.g. we are supposed to grasp right away what is "a part" and to have no difficulty imagining an entity without parts. He describes semantics of basic geometric notions in terms of a slightly refined non-verbal everyday experience.

Second, for a possible discoverer and a great practitioner of the axiomatic method, he is strangely oblivious about some one-step logical implications of his definitions. If we take the "extremity" (I will also use the modern term "boundary") of a solid ball, it must be a surface, namely, sphere. Now, the boundary of this surface is not a line, contrary to Book I, 6, because it is empty!

Thus, Euclid misses a great opportunity here: if he stated the principle

$$
\text { "The extremity of an extremity is empty," }
$$

he could be considered as the discoverer of the

BASIC EQUATION OF HOMOLOGICAL ALGEBRA:

$$
d^{2}=0
$$

For a historian of culture, the reason for this strange blindness is obvious: "emptiness" and "zero" as legitimate notions, solidly built into systematic scientific thinking, appear much later.

Even contemporary thought periodically betrays persistent intellectual uneasiness regarding emptiness: compare the reification of "Nothing" commonly performed in philosophical texts of many schools and various versions of "vacuum state" in the quantum field theory.

Skipping two milleniums, we turn now to Leibniz. The following excerpt from Mand, p. 405, introduces a modern algebraic idea related to our further discussion of fractional dimensions:

"[...] the idea of fractional integro-differentiation [...] occurred to Leibniz, as soon as he has developed his version of calculus and invented the notations $d^{k} F / d x^{k}$ and $(d / d x)^{k} F$. In free translation of Leibniz's letter to de l'Hôpital dated September 30, $1695[\ldots]:$

Johann Bernoulli seems to have told you of my having mentioned to him a marvelous analogy which makes it possible to say in a way that successive differentials are in geometric progression. One can ask what would be a differential having as its exponent a fraction. [...] Although this seems removed from Geometry, which does not yet know of such fractional exponents, it appears that one day these paradoxes will yield useful consequences, since there is hardly a paradox without utility."

I read the first part of this quotation as a reference to what later became known as Taylor series: in the formula $f(x+d x)=\sum_{n=0}^{\infty} \frac{f^{(n)}(x)}{n !}(d x)^{n}$ the consecutive terms are "successive differentials in geometric progression". 
By extension, I will interpret Leibniz's quest as follows: "Make sense of the formal expression $f(x)(d x)^{s}$, with arbitrary rational, or real, or even complex value of $s$ (we may add $p$-adic values as well).

Nowadays it is easy to give a Bourbaki-style answer to this quest. Let $M$ be a differentiable manifold, $s$ an arbitrary complex number. Then we can construct:

(i) A rank one complex vector bundle $V_{s}$ of $s$-densities on $M$, which is trivialized over each coordinate neighborhood $\left(x_{i}\right)$ and for which the transition multiplier from $\left(y_{j}\right)$ to $\left(x_{i}\right)$ is

$$
\left|\operatorname{det}\left(\partial x_{i} / \partial y_{j}\right)\right|^{s}
$$

(ii) Its sections locally can be written as $h(x)|d x|^{s}$. Spaces of sections $W_{s}$ with various integrability, differentiability, growth, etc., conditions are called $s$-densities.

(iii) (Some) sections of $V_{1}$ are measures, so that they can be integrated on $M$.

(iv) This produces a scalar product on (various spaces of) densities:

$$
W_{s} \times W_{1-s} \rightarrow \mathbf{C}: \quad\left(f|d x|^{s}, g|d x|^{1-s}\right) \mapsto \int_{M} f g|d x| .
$$

Roughly speaking, subsets $N \subset M$ of (normalized) fractional dimensions $s \operatorname{dim} V$ $\in \mathbf{R}$ appear when we learn that $W_{s}$ can be integrated along them, as $W_{1}$ can be integrated along $M$. Various ramifications and amplifications of this idea will be reviewed below.

Euclid and Leibniz were chosen to represent in this Introduction respectively right- and left-brain modes of thinking, characteristic traits of which became well known after popularizations of Roger Sperry's work on brain asymmetry (Nobel Prize 1981). In mathematical thinking, they roughly correspond to the dichotomies Geometry/Algebra, Vision/Formal Deduction, etc. As I have written elsewhere ( Ma4 $)$ :

"A natural or acquired predilection towards geometric or algebraic thinking and respective mental objects is often expressed in strong pronouncements, like Hermann Weyl's exorcising 'the devil of abstract algebra', who allegedly struggles with 'the angel of geometry' for the soul of each mathematical theory. (One is reminded of an even more sweeping truth: 'L'enfer - c'est les autres.')

"Actually, the most fascinating thing about Algebra and Geometry is the way they struggle to help each other to emerge from the chaos of non-being, from those dark depths of subconscious where all roots of intellectual creativity reside. What one 'sees' geometrically must be conveyed to others in words and symbols. If the resulting text can never be a perfect vehicle for the private and personal vision, the vision itself can never achieve maturity without being subject to the test of written speech. The latter is, after all, the basis of the social existence of mathematics.

"A skillful use of the interpretative algebraic language possesses also a definite therapeutic quality. It allows one to fight the obsession which often accompanies contemplation of the enigmatic Rorschach's blots of one's private imagination.

"When a significant new unit of meaning (technically, a mathematical definition or a mathematical fact) emerges from such a struggle, the mathematical community spends some time elaborating all conceivable implications of this discovery. (As an example, imagine the development of the idea of a continuous function, or a Riemannian metric, or a structure sheaf.) Interiorized, these implications prepare new firm ground for further flights of imagination and more often than not reveal the limitations of the initial formalization of the geometric intuition. Gradually the discrepancy between the limited scope of this unit of meaning and our newly 
educated and enhanced geometric vision becomes glaring, and the cycle repeats itself."

This all-pervasive Left/Right dichotomy has also a distinctive social dimension, which recently led to the juxtaposition of "Culture of the Word" and "Culture of the Image". The society we live in becomes more and more dominated by mass media/computer generated images (to which visual representations of "fractals", sets of non-integer dimension, marginally belong). Paradoxically, this technologically driven evolution away from "logocentrism", often associated with modernity and progress, by relying heavily upon right brain mental faculties, projects us directly into dangerously archaic states of collective consciousness.

0.2. Plan of the paper. The first section presents several contexts in which one can define dimension transcending the core intuition of "number of independent degrees of freedom": Hausdorff-Besicovich dimension, dimensional regularization, Murray-von Neumann dimension.

The second section introduces dimension in supergeometry and discusses the question: what is dimension of Spec $\mathbf{Z}$ ?

The third section is devoted to the spectrum of dimensions Leibniz style arising in the theory of modular forms.

Finally, in the fourth section I review some recent constructions introducing fractional dimensions in homological algebra. Although their source was Mirror Symmetry, I have chosen to present a theorem due to Polishchuk which puts this subject in the context of non-commutative geometry and Real Multiplication program for quantum tori. With some reluctance, I decided to omit another fascinating development, which can also be related to Mirror Symmetry, "motives of fractional weights" (cf. And]).

\section{ACKNOWLEDGMENTS}

This paper owes its existence to Susan Friedlander, who also made useful comments on a preliminary version and did some linguistic editing. After my talk at the AMS meeting, Edward Frenkel reminded me about my old report Ma1. Matilde Marcolli read the first draft of this paper and suggested the inclusion of a discussion of Connes' notion of dimension spectrum and Lapidus' related notion of complex dimensions. She has also written detailed instructions to that effect. The whole $\S 2$ owes its existence to them and provides an additional motivation for my presentation of the Lewis-Zagier theory in $\S 3$. A. Beilinson and A. Polishchuk drew my attention to MacPherson's earlier explanation of perverse sheaves on simplicial complexes.

\section{§1. Fractional Dimensions: a COncise Collector's guide}

1.1. Hausdorff-Besicovich dimension. The scene here is a metric space $M$. What is defined and counted: $H B$-dimension of an arbitrary subset $S \subset M$ with a compact closure.

Strategy of counting:

(i) Establish that a Euclidean $d$-dimensional ball $B_{\rho}$ of radius $\rho$ has volume

$$
\operatorname{vol}_{d}\left(B_{\rho}\right)=\frac{\Gamma(1 / 2)^{d}}{\Gamma(1+d / 2)} \rho^{d} .
$$

Here $d$ is a natural number. 
(ii) Declare that a $d$-dimensional ball $B_{\rho}$ of radius $\rho$ has volume given by the same formula

$$
\operatorname{vol}_{d}\left(B_{\rho}\right)=\frac{\Gamma(1 / 2)^{d}}{\Gamma(1+d / 2)} \rho^{d}
$$

for any real $d$.

(iii) Cover $S$ by a finite number of balls of radii $\rho_{m}$.

(iv) Make a tentative count: measure $S$ as if it were $d$-dimensional for some $d$ :

$$
v_{d}(S):=\lim _{\rho \rightarrow 0} \inf _{\rho_{m}<\rho} \sum_{m} \operatorname{vol}_{d}\left(B_{\rho_{m}}\right) .
$$

(v) Grand Finale: There exists $D$ such that $v_{d}(S)=0$ for $d>D$ and $v_{d}(S)=\infty$ for $d<D$.

This $D$ is declared to be the HB-dimension of $S$.

A set of non-integral Hausdorff-Besicovich dimension is called a fractal (B. Mandelbrot).

The general existence of $D$ is a remarkable mathematical phenomenon, akin to those that appear in the description of phase transitions and critical exponents in physics. To get a feeling how it works, consider first the simple example: how do we see that $[0,1]$ isometrically embedded in $M$ has dimension one? Basically, we can cover $[0,1]$ by $N$ closed balls of diameter $\rho=N^{-1}$ centered at points of $S$. In a tentative count assuming dimension $d$, we get approximate volume $c_{d} N \cdot(1 / 2 N)^{d}$ which tends to 0 (resp. to $\infty$ ) with $N \rightarrow \infty$ when $d>1$ (resp. $d<1$ ). Hence $D=1$.

A similar counting (which can be easily remade into a formal proof) shows that the classical Cantor subset $C \subset[0,1]$ has dimension $\log 2 / \log 3$, so it is a fractal.

A further remark: the constant involving gamma-factors in the formula (1.1) for $\operatorname{vol}_{d}\left(B_{\rho}\right)$ does not influence the value of $D$. However, some $S$ of HB-dimension $D$ may have a definite value of $v_{D}(S)$, that is, be HB-measurable. The value of this volume will then depend on the normalization. Moreover, one can slightly change the scene and work with, say, differentiable ambient manifolds $M$ and $s$-densities in place of volumes of balls. This leads to the picture of integration of densities I referred to in the Introduction.

I do not know whether some subsets $S$ are so topologically "good" as to deserve the name of $D$-dimensional manifolds. Is there a (co)homology theory with geometric flavor involving such fractional dimensional sets?

1.2. Fractional dimensions in search of a space: dimensional regularization of path integrals. Here is a very brief background; for details, see $[\mathrm{Kr}$, $\mathrm{CoKr}$ and references therein.

Correlators in a quantum field theory are given heuristically by Feynmann path integrals. A perturbative approach to defining such an integral produces a formal series whose terms are indexed by Feynman graphs and are familiar finite dimensional integrals.

However, each term of such a formal series usually diverges. A procedure that regularizes it by subtracting appropriate infinites is called regularization/renormalization. Each such procedure involves a choice of a certain parameter, a value of a mass scale, of an interaction constant, etc., which is then made variable in such a way that integrals become finite at "non-physical" values of this parameter. A 
study of their analytic behavior near the physical point then furnishes concrete divergent terms, or counterterms, which are subtracted.

Dimensional regularization is a specific regularization procedure which replaces the physical dimension of space time (4 in the case of a scalar non-stringy field theory) by a complex variable $D$ varying in a small neighborhood of 4 .

Instead of extrapolating volumes of balls to non-integral $D$, one extrapolates here the values of a Gaussian integral:

$$
\int e^{-\lambda}|k|^{2} d^{D} k=\left(\frac{\pi}{\lambda}\right)^{D / 2} .
$$

However, no explicit sets making geometric sense of (1.2) occur in the theory.

The germ of the $D$-plane near $D=4$ then becomes the base of a flat connection with an irregular singularity. The regularization procedure can be identified with taking the regular part of a Birkhoff decomposition (A. Connes - D. Kreimer).

If one wishes to think of spaces of such complex dimensions, one should probably turn to non-commutative geometry: cf. subsections 1.4 and 2.4 below.

1.3. Murray-von Neumann factors. Here the introductory scene unfolds in a linear space $M$ (say, over $\mathbf{C}$ ). What is counted: dimension of a linear subspace $L \subset M$.

How fractional dimensions occur: if the usual linear dimensions of $L$ and $M$ are both infinite, it might happen nevertheless that a relative $\operatorname{dimension} \frac{\operatorname{dim} L}{\operatorname{dim} M}$ makes sense and is finite.

To be more precise, we must first rewrite the finite dimensional theory stressing the matrix algebra $E_{M}:=$ End $M$ in place of $M$ itself. $L$.

Replace $L$ by (the conjugacy class of) projector(s) $p_{L} \in E_{M}: p_{L}^{2}=p_{L}, p_{L}(M)=$

Construct a (normalized) trace functional $t r: E_{M} \rightarrow$ C. A natural normalization condition here is $\operatorname{tr}\left(i d_{M}\right)=1$. Another normalization condition might be $\operatorname{tr}\left(p_{L}\right)=1$ where $L$ is a subspace having no proper subspaces.

Define the (fractional) dimension of $L$ as $\operatorname{tr}\left(p_{L}\right)$.

Murray and von Neumann pass from this elementary picture to the following setting: take for $L$ a Hilbert space; define $W$-algebras acting on $L$ as algebras of bounded operators with abstract properties similar to that of $E_{M}$.

For a reasonable class of such algebras (factors), study the spectrum of fractional dimensions: values of (possibly normalized) trace functionals $t$ on the equivalence classes of projections $t\left(p_{L}\right)$ (projections are selfadjoint projectors).

The remarkably beautiful Murray and von Neumann classification theorem then says that the normalized spectra of dimensions can be exactly of five types:

$\mathrm{I}_{n}:\{1, \ldots, n\}$.

$\mathrm{I}_{\infty}:\{1, \ldots, \infty\}$.

$\mathrm{II}_{1}:[0,1]$

$\mathrm{II}_{\infty}:[0,+\infty]$

III: $\{0,+\infty\}$.

1.4. Non-commutative Geometry. A vast project (actually, a vast building site) of Non-commutative Geometry is dominated by two different motivations. A powerful stimulus is furnished by physics: quantum theory replaces commuting observables by generally non-commuting operators. 
Another motivation comes from mathematics, and its conception has a definitely algebraic origin.

It is a fact that the study of all more or less rigid geometric structures (excluding perhaps homotopical topology) is based on a notion of (local) functions on the respective space, which in turn evolved from the idea of coordinates that revolutionized mathematics. After Grothendieck, this idea acquired such a universality that, for example, any commutative ring $A$ now comes equipped with a space on which $A$ is realized as an algebra of functions: the scheme Spec $A$.

In non-commutative geometry we allow ourselves to think about non-commutative rings (but also about much more general structures, eventually categories, polycategories, etc.) as coordinate rings of a "space" (resp. sheaves, etc., on this space). What constitutes the "existential characteristics" of such a space, what algebraic constructions reveal its geometry, how to think about them orderly and creatively, - these are the challenges that fascinate many practitioners in this field.

One device for efficient training of our geometric intuition is the consistent study of commutative geometry from a non-commutative perspective. In particular, an important role is played by certain spaces which appear as "bad quotients" of some perfectly sane commutative manifolds. A standard example is furnished by the space of leaves of a foliation: such a space is well defined as a set but generally has a very bad topology. Alain Connes in $\mathrm{Co} 2$ sketched the general philosophy and provided a ghost of beautiful examples of such situations. The starting point in many cases is the following prescription for constructing a non-commutative ring describing a bad quotient $M / \mathcal{R}$ : take a function ring $A$ of $M$ and replace it by a certain crossed product $A \rtimes \mathcal{R}$.

Probably the simplest example of a bad quotient is provided not by a foliation, but by the trivial action of a, say, finite group $G$ on a point. The quotient $S:=$ $\{p t\} / G$ with respect to such an action is represented by the group algebra $A_{S}:=$ $\mathbf{C}[G]$. We imagine $A_{S}$ as "an algebra of functions on a non-commutative space $S^{n c}$ " and apply to it the generic dictionary of the Algebra $\Leftrightarrow$ Geometry correspondence:

a measure on $S^{n c}:=$ a linear functional on $A_{S}$;

a vector bundle on $S^{n c}:=$ a projective module over $A_{S} \ldots$ etc.

Fractional dimensions of von Neumann and Murray settings reappear in this context, with potentially very different geometric interpretation.

At this point, it's worth stressing the difference between classical fractals and such non-commutative spaces: the former are embedded in "good" spaces, the latter are their projections. Living in a Platonic cave, we have more psychological difficulties in recognizing and describing these projections.

1.5. Digression on databases. A large database $B$ with links can be imagined as a vast metric space. We can envision its graph approximation: pages $\Rightarrow$ vertices, links $\Rightarrow$ edges. Metric can be defined by the condition that a link has length one, or else: length of a link is the relative number of hits.

Approximate dimension $d$ then can be introduced: a (weighted) number of pages accessible in $\leq R$ links is approximately $c R^{d}$.

Some experimental work with actual databases produces definitely non-integer dimensions $(\underline{\mathrm{Ma}}])$.

Databases are used for search of information. A search in $B$ usually produces a "bad subset" $S$ in $B$, for example, all contexts of a given word. 
If the database $B$ registers results of scientific observations (Human Genome project, cosmology), what we would like to get from it is instead (a fragment of) $a$ new scientific theory.

Arguably, such a theory is "a bad quotient" rather than a bad subset of $B$.

Imagine all Darwin's observations registered as a raw database, and imagine how evolutionary theory might have been deduced from it: drawing bold analogies and performing a drastic compression. Arguably, both procedures are better modeled by "bad equivalence relations" rather than by contextual search.

\section{§2. Exotic Dimensions}

Up to now, I mostly used the word "dimension" in the sense "the number of degrees of freedom" (appropriately counted). In the title of this section, "exotic dimension" means "an unusual degree of freedom" and is applied, first, to odd dimensions of supergeometry and, second, to the arithmetical line Spec Z. Subsections 2.1 and 2.2 can be read as a post-scriptum to the review written twenty years ago: see Ma1] and At].

Starting with 2.3, we explain the notion that (doubled real parts of) zeroes and poles of various zeta-functions of geometric origin $Z(X, s)$ can be viewed as a "dimension spectrum". One source of this notion is the algebraic/arithmetic geometry of varieties over finite or number fields. Another source, and the term "dimension spectrum", is Connes' work on non-commutative Riemannian geometry (cf. [Co2, VI, IV.3. $\gamma$ ) and a closely related work of M. Lapidus and collaborators in fractal geometry ([LaPo, [LavF1], [LavF2]).

2.1. Supergeometry. Coordinate rings in supergeometry are $\mathbf{Z}_{2}$-graded and supercommutative: we have $f g=(-1)^{|f||g|} g f$ where $|f|$ denotes the parity of $f$. This Koszul sign rule applies generally in all algebraic constructions. The algebraic coordinate ring of an affine space $A_{K}^{m \mid n}$ over a field $K$ is $K\left[x_{1}, \ldots, x_{m} ; \xi_{1}, \ldots, \xi_{n}\right]$ where $x$ 's are even and $\xi$ 's are odd. Its (super)dimension is denoted $m \mid n$.

Since odd functions are nilpotents, the usual intuition tells us that, say, $A_{K}^{0 \mid n}$ can only be imagined as an "infinitesimal neighborhood" of the point $S p e c K$. This seemingly contradicts our desire to see this superspace as a "pure odd manifold". A slightly more sophisticated reasoning will convince us that $A_{K}^{0 \mid n}$ does have the defining property of a manifold: its cotangent sheaf (universal target of odd derivations) is free, again due to the Koszul sign rule.

The basics of all geometric theories can be readily extended to superspaces. Deeper results also abound, in particular, the Lie-Cartan classification of simple Lie algebras is extended in a very interesting way.

The physical motivation for introducing odd coordinates was Fermi statistics for elementary particles and the conjecture that laws of quantum field theory include supersymmetry of appropriate Lagrangians.

\subsection{What is the value of dimension of $\operatorname{Spec} \mathbf{Z}$ ?}

Answer 1: $\operatorname{dim} \operatorname{Spec} \mathbf{Z}=1$. This is the common wisdom. Formally, one is the value of Krull dimension of $\mathbf{Z}$, maximal length of a chain of embedded prime ideals. Krull dimension can be viewed as a natural algebraization of Euclid's inductive definition of dimension. From this perspective, primes $p$ are zero-dimensional points of $\operatorname{Spec} \mathbf{Z}$, images of geometric points $\operatorname{Spec} \mathbf{F}_{p} \rightarrow \operatorname{Spec} \mathbf{Z}$. 
Answer 2: $\operatorname{dim} \operatorname{Spec} \mathbf{Z}=3$. One can argue, however, that $\operatorname{Spec} \mathbf{F}_{p}$ is not zerodimensional, because its fundamental group, $\operatorname{Gal} \overline{\mathbf{F}}_{p} / \mathbf{F}_{p}$, is the same as (the completed) fundamental group of the circle. Images of $\operatorname{Spec} \mathbf{F}_{p} \rightarrow \operatorname{Spec} \mathbf{Z}$ should be then visualized as loops in the space $\operatorname{Spec} \mathbf{Z}$, and one can define their linking numbers which turn out to be related to the reciprocity laws and Legendre and Rédei symbols. For this reason, Spec $\mathbf{Z}$ "must" be three-dimensional: cf. a review in Mor and references therein.

More systematically, in étale topology of $\operatorname{Spec} \mathbf{Z}$ one can observe 3-dimensional Poincaré duality: see Maz.

Before the advent of étale topology and even schemes, Selberg made the remarkable discovery that lengths of closed geodesics in hyperbolic spaces behave like primes: Selberg's zeta functions are close relatives of Riemann's zeta. This gives additional weight to the idea that primes "are" loops.

Answer 3: $\operatorname{dim} \operatorname{Spec} \mathbf{Z}=\infty$ ? This guess involves the conjectural existence of a geometrical world defined over "an absolute point" $\operatorname{Spec} \mathbf{F}_{1}$ where $\mathbf{F}_{1}$ is a mythical field with one element. For some insights about this world, see [Ti], Sm1, [Sm2], KapSm, Ma2, Sou].

In particular, Soulé in $\left[\mathrm{Sou}\right.$ defined a category of varieties over $\operatorname{Spec} \mathbf{F}_{1}$ which presumably should be thought of as varieties of finite type. This category does not contain Spec $\mathbf{Z}$. In fact, objects $V$ of this category are defined via properties of their purported base extensions $V \times_{\mathbf{F}_{1}} \mathbf{Z}$, whereas $\mathbf{Z} \times_{\mathbf{F}_{1}} \mathbf{Z}$ remains tantalizingly elusive.

And if $\operatorname{Spec} \mathbf{Z}$ is not a finite type object, it can hardly have a finite dimension.

2.2.1. Summary. The discussion so far can be interpreted as leading to the following conclusion: not only the arithmetical degree of freedom of $\operatorname{Spec} \mathbf{Z}$ is exotic but the value of the respective dimension is not just a real number or infinity but a new entity which deserves special attention. We probe the arithmetical degree of freedom by studying its interaction with "geometric" degrees of freedom, in particular, studying algebraic varieties over finite and number fields and rings.

For this reason, the whole arithmetic geometry, in particular, Arakelov's insights and their subsequent development, Deninger's program [De1-De4, relations with non-commutative geometry as in Co5, ConsMar, and Haran's visions $\mathrm{Ha}$, will bear upon our future enlightened decision about what $\operatorname{dim} \operatorname{Spec} \mathbf{Z}$ actually is. Below we will briefly relate the role of zeta functions from this perspective.

2.3. Zeta functions and weights in arithmetic geometry. Let $C$ be a smooth irreducible projective algebraic curve defined over $\mathbf{F}_{q}$. Its zeta function can be defined by a Dirichlet series and an Euler product, in perfect analogy with Riemann zeta:

$$
Z(C, s)=\sum_{a} \frac{1}{N(a)^{s}}=\prod_{x} \frac{1}{1-N(x)^{-s}} .
$$

Here $x$ runs over closed points of $C$, playing the role of primes, and $a$ runs over effective cycles rational over $\mathbf{F}_{q}$. It is an elementary exercise to rewrite (2.1) in terms of a generating function involving all numbers card $V\left(\mathbf{F}_{q^{f}}\right)$. The latter can be interpreted as the numbers of fixed points of powers of the Frobenius operator Fr acting upon $C\left(\overline{\mathbf{F}}_{q}\right)$. A. Weil's remarkable insight consisted of postulating the existence of a cohomology theory and a Lefschetz type formula counting these fixed 
points, proving it for curves, and conjecturally extending it to general projective manifolds over finite fields. The result for curves reads

$$
\begin{aligned}
Z(C, s) & =\prod_{w=0}^{2} \operatorname{det}\left(\left(\operatorname{Id}-F r \cdot q^{-s}\right) \mid H^{w}(C)\right)^{(-1)^{w-1}} \\
& =\prod_{w=0}^{2} Z\left(h^{w}(C), s\right)^{(-1)^{w-1}} .
\end{aligned}
$$

More generally, for a smooth irreducible projective manifold $V$ defined over $\mathbf{F}_{q}$ we can define the zeta function by a formula similar to (2.1) and, after Grothendieck and Deligne, prove the formula

$$
\begin{aligned}
Z(V, s) & =\prod_{w=0}^{2 \operatorname{dim} V} \operatorname{det}\left(\left(\mathrm{Id}-F r \cdot q^{-s}\right) \mid H^{w}(V)\right)^{(-1)^{w-1}} \\
& =\prod_{w=0}^{2 \operatorname{dim} V} Z\left(h^{w}(V), s\right)^{(-1)^{w-1}} .
\end{aligned}
$$

Here $H^{w}(V)$ denotes étale cohomology of weight $w$, whereas $h^{w}(V)$ refers to the motivic piece of $V$ of weight $w$ which is a kind of universal cohomology. According to the Riemann-Weil conjecture proved by Deligne, the roots $\rho$ of $Z\left(h^{w}(V), s\right)$ lie on the vertical line $\operatorname{Re} \rho=\frac{w}{2}$.

Thus we can read off the spectrum of dimensions in which $V$ "manifests itself" non-trivially (i.e. by having a non-trivial cohomology group) by

(i) Counting fixed points of Frobenius on $V\left(\overline{\mathbf{F}}_{q}\right)$.

(ii) Looking at the zeroes and poles of the zeta function produced by this count.

Note also that the expression occurring in (2.3) very naturally appears in the supergeometry of the total cohomology space $H^{*}(V)$ graded by the parity of weight: this is simply the inverse superdeterminant of the operator Id $-F r \cdot q^{-s}$. More generally, quantum cohomology introduces a quite non-trivial and non-linear structure on this cohomology considered as a supermanifold and not just $\mathbf{Z}_{2}$-graded linear space. This is how supergeometry enters classical mathematics through the back door.

Let us return now to $\operatorname{Spec} \mathbf{Z}$. Deninger in De1] suggested writing the following analog of (2.2) for Riemann's zeta multiplied by the $\Gamma$-factor interpreted as the Euler factor at arithmetic infinity:

$$
\begin{gathered}
Z(\overline{\operatorname{Spec} \mathbf{Z}}, s):=2^{-1 / 2} \pi^{-s / 2} \Gamma\left(\frac{s}{2}\right) \zeta(s)=\frac{\prod_{\rho} \frac{s-\rho}{2 \pi}}{\frac{s}{2 \pi} \frac{s-1}{2 \pi}} \\
=(?) \prod_{\omega=0}^{2} \operatorname{DET}\left(\frac{s \cdot \operatorname{Id}-\Phi}{2 \pi} \mid H_{?}^{\omega}(\overline{\operatorname{Spec} \mathbf{Z}})\right)^{(-1)^{w-1}} .
\end{gathered}
$$

Here the notation $\prod_{\rho}$ as well as the conjectural DET refers to the "zeta regularized" infinite products which are defined by

$$
\prod_{i} \lambda_{i}:=\exp \left(-\left.\frac{d}{d z} \sum_{i} \lambda_{i}^{-z}\right|_{z=0}\right) .
$$

The second equality sign in (2.4) is a theorem, whereas the last equality sign expresses a conjecture about the existence of some cohomology theory and a Frobenius 
type operator on it. In fact, $\Phi$ should be considered rather as a logarithm of Frobenius: the direct comparison must be made between (2.4) and (2.2) rewritten with the help of another identity:

$$
1-\mu q^{-s}=\prod_{\alpha: q^{\alpha}=\mu} \frac{\log q}{2 \pi i}(s-\alpha) .
$$

Finally, to study the interaction between $\operatorname{Spec} \mathbf{Z}$ and geometric dimensions, one considers zeta functions of schemes of finite type over $\operatorname{Spec} \mathbf{Z}$ which are, say, models of smooth projective manifolds over number fields, or even motives of this type. A series of partial results and sweeping conjectures suggests a similar picture for such zetas, with real parts of zeroes/poles producing a spectrum of "absolute weights" of arithmetical schemes.

For more details, see a discussion in Ma3 and more recent speculations on the nature of geometry behind the Frobenius $\Phi$ and Deninger's cohomology. In particular, De3], De4 postulate existence of dynamical systems underlying this geometry, whereas ConsMar] introduces non-commutative spaces responsible for $\Gamma$-factors for curves, and Connes in $\mathrm{Co5}$ outlines an approach to the Riemann hypothesis by way of non-commutative geometry.

2.4. Dimension spectra of spectral triples. Zeta functions of another kind arise in the context of Connes spectral triples. This is a reformulation of basic data of Riemannian geometry which can be directly generalized to the non-commutative case: cf. $\mathrm{Co} 3$, $\mathrm{CoMos}$.

We will now briefly describe the constructions relevant to our discussion of dimension spectra.

A spectral triple consists of data $(A, H, D)$, where $A$ is a $*$-subalgebra of bounded operators on a Hilbert space $H$, whereas $D$ is an unbounded self-adjoint operator on $H$, with compact resolvent. The relevant compatibility condition between $A$ and $D$ reads as follows: commutators $[a, D]$ are bounded for all $a \in A$.

The prototype of such a structure is the triple $\left(C^{\infty}(X), L^{2}(S), D\right)$ associated to a compact spin manifold $X$, where $S$ is the spinor bundle and $D$ the Dirac operator. Examples of spectral triples in genuinely non-commutative cases have been constructed in the context of quantum groups ([Co6], DaLSSV] $)$, in arithmetic geometry (ConsMar $)$, and as a proposed geometric model of elementary particle physics ( $\mathrm{Co} 4)$.

Spectral triples of finite summability degree (that is, where $|D|^{z}$ is trace class for some $z$ ) provide the stage for a universal local index formula (Connes-Moscovici) for the cyclic cohomology Chern character associated to the index problem

$$
\operatorname{Ind}_{D}: K_{*}(A) \rightarrow \mathbf{Z} .
$$

The local index formula is given in terms of the Wodzicki residue on the algebra of pseudodifferential operators of $(A, H, D)$. Extending the Wodzicki residue to this context amounts to extending the Dixmier trace to operators of the form $b|D|^{-z}$, where $b$ is in the algebra $B$ generated by the elements $\delta^{n}(a)$, for $\delta(a)=[|D|, a]$ and $a \in A$.

It is here that the notion of dimension spectrum naturally enters the scene. Namely, the fact that the Wodzicki residue continues to make sense and defines a trace depends upon the properties of a family of zeta functions associated to the 
spectral triple $(A, H, D)$,

$$
\zeta_{b}(z)=\operatorname{Tr}\left(b|D|^{-z}\right), \quad b \in B .
$$

In particular, the dimension spectrum $\Sigma \subset \mathbf{C}$ is the minimal subset such that, for all $b \in B$, the zeta function $\zeta_{b}$ extends holomorphically to $\mathbf{C} \backslash \Sigma$.

If this subset is discrete and singularities of $\zeta_{b}(s)$ are simple poles, one can extend the Dixmier trace by the formula

$$
\int b:=\operatorname{Res}_{z=0} \operatorname{Tr}\left(b|D|^{-z}\right) .
$$

The case of the Connes-Moscovici local index formula where multiplicities appear in the dimension spectrum is treated by applying renormalization group techniques ( $\mathrm{Co} 3, \mathrm{CoMos}$ ).

In what sense can one think of $\Sigma$ as a "set of dimensions" for the non-commutative manifold $(A, H, D)$ ?

In the context of this paper, a comparison with (2.2)-(2.4) may help the reader. Connes includes in the dimension spectrum only singularities of his zetas. It seems that they should be compared with zeroes of (the numerator of) (2.2), (2.4), that is, with poles of the inverted arithmetical zeta, corresponding to the motivic part of odd weight. This inversion corresponds in supergeometry to the parity change, which might explain the remark made in section II.1, p. 205, of CoMos] about advantages of treating the odd case.

If $(A, H, D)$ comes from a $p$-dimensional compact spin manifold, the dimension spectrum of it is contained in $\{n \in \mathbf{Z} \mid n \leq p\}$ ([CoMos, p. 211), and the relevant singularities of zeta are simple poles.

One can associate spectral triples to certain fractal sets and calculate their spectra. Even more straightforward constructions can be given for special fractal sets like fractal strings or generalized fractal strings of Lapidus and van Frankenhuysen; cf. [LavF2, Ch. 3.

Sometimes it turns out that the whole spectrum lies on the line $\operatorname{Re} s=D_{0}$, where $D_{0}$ is the Hausdorff-Besicovich dimension. This again agrees with the arithmetic geometry case: cf. our discussion of Riemann hypothesis after formula (2.3). The extra factor $1 / 2$ appearing there is nicely explained by a peculiar normalization: roughly speaking, in algebraic geometry the dimension of the complex line is 1 , so the dimension of the real line should be $1 / 2$.

The dimension spectrum has the expected behavior with respect to the product of spectral triples. In arithmetic geometry, similar arguments lead to the highly speculative picture of Kurokawa's tensor product of zeta functions which presumably reflects a direct product operation over "the absolute point" $\mathbf{F}_{1}$; cf. [Ma3].

The question of possible relations between dimension spectra, the local index formula, and dimensional regularization arise naturally in CoMar2, where the Connes-Kreimer theory of perturbative renormalization is reformulated as a Riemann-Hilbert problem for a certain class of flat connections with irregular singularities (equisingular). In fact, the universal singular frame, which produces "universal counterterms" for all the renormalizable field theories, has exactly the same rational coefficients that appear in the local index formula of Connes-Moscovici. This suggests the possibility that the missing geometry underlying dimensional regularization may be found in non-commutative spaces whose dimension spectrum lies fully off the real line. 
The fact that Spec $\mathbf{Z}$ "manifests itself non-trivially" both in dimensions one and three raises the possibility that it can be connected with the dimension spectrum of the (as yet conjectural) spectral triple for a non-commutative space such as the adelic quotient considered by Connes in the spectral realization of the zeros of zeta (Co5]), which is in turn the space of commensurability classes of 1-dimensional Q-lattices of CoMar1.

In the next section I will review some recent results due to J. Lewis and D. Zagier providing a geometric scene for the interpretation of dimension spectrum of a Selberg zeta function. Although spectral triples do not explicitly appear there, the spirit is very similar.

\section{§3. MOdular FORMS AND Weights}

3.1. Classical modular forms. A classical modular form of weight $w+2$ with respect to a subgroup $\Gamma \subset S L(2, \mathbf{Z})$ is a meromorphic function $f$ on upper halfplane $H$ satisfying

$$
f\left(\frac{a z+b}{c z+d}\right)=f(z)(c z+d)^{w+2}
$$

for all fractional linear transformations from $\Gamma$. Let us assume that $w$ is an even integer; then (3.1) means that the formal expression $f(z)(d z)^{(w+2) / 2}$ is $\Gamma$-invariant and hence is (the lift of) a Leibniz's higher differential on $X_{\Gamma}=\Gamma \backslash H$.

One can also rewrite (3.1) differently by looking at the universal elliptic curve $E_{\Gamma} \rightarrow X_{\Gamma}$ and the Kuga-Sato variety which is (a compactification of) $E_{\Gamma}^{(w)}:=$ $E_{\Gamma} \times_{X_{\Gamma}} \cdots \times_{X_{\Gamma}} E_{\Gamma}$ ( $w$ times $)$. Namely, $E_{\Gamma}^{(w)}$ is the quotient of $\mathbf{C}^{w} \times H$ with respect to the group $\Gamma^{(w)}:=\mathbf{Z}^{2 w} \rtimes \Gamma$ where the group of shifts $\mathbf{Z}^{2 w}$ acts upon fibers by

$$
\left(\left(t_{k}\right), z\right) \mapsto\left(\left(t_{k}+m_{k} z+n_{k}\right), z\right),
$$

whereas $\Gamma$ acts by

$$
\left(\left(t_{k}\right), z\right) \mapsto\left(\left(\frac{t_{k}}{c z+d}\right), \frac{a z+b}{c z+d}\right) .
$$

In this notation, (3.1) means that the meromorphic volume form $f(z) d z \wedge d t_{1} \wedge$ $\cdots \wedge d t_{w}$ comes from a volume form $F$ on $E_{\Gamma}^{(w)}$. Thus $w+1$ is the dimension of a classical space. Periods of cusp forms $f$, which are by definition integrals $\int_{0}^{i \infty} f(z) z^{k} d z, 0 \leq k \leq w$, can also be expressed as integrals of $F$ over appropriate cycles in $E_{\Gamma}^{(w)}$.

Below I will describe modular forms of fractional weight of two types:

(i) Serre's forms with $p$-adic weights,

(ii) Lewis-Zagier period functions with complex weights.

3.2. Serre's modular forms of $p$-adic weight. Here we will consider the case $\Gamma=S L(2, \mathbf{Z})$. Let a modular form be finite at all cusps, in particular given by its Fourier series of the form $\sum_{n=0}^{\infty} a_{n} q^{n}, q:=e^{2 \pi i z}$. Former $w+2$ is now denoted $k$ and also called a weight.

3.2.1. Definition. A p-adic modular form is a formal series

$$
f=\sum_{n=0}^{\infty} a_{n} q^{n}, \quad a_{n} \in \mathbf{Q}_{p},
$$


such that there exists a sequence of modular forms $f_{i}$ of weights $k_{i}$ with rational coefficients p-adically converging to $f$ (in the sense of uniform convergence of coefficients).

3.2. Theorem. The p-adic limit

$$
k=k(f):=\lim _{i \rightarrow \infty} k_{i} \in \lim _{m \rightarrow \infty} \mathbf{Z} /(p-1) p^{m} \mathbf{Z}=\mathbf{Z}_{p} \times \mathbf{Z} /(p-1) \mathbf{Z}
$$

exists and depends only on $f$. It is called the p-adic weight of $f$.

For a proof, see $\mathrm{Se}$. This theorem enhances some constructions which initially appeared in the theory of $p$-adic interpolation of $L$-series; see also $\mathrm{Ka}$ for a broader context.

Can one make sense of a $p$-adic limit of Kuga spaces $E_{\Gamma}^{\left(k_{i}-1\right)}$ or, rather, appropriate motives?

3.3. Modular forms at the boundary. Quotients $X_{\Gamma}=\Gamma \backslash H$ are non-compact modular curves. In algebraic geometry, they are compactified by adding cusps $\Gamma \backslash \mathbf{P}^{1}(\mathbf{Q})$. Recently in several papers it was suggested that one should consider as well the "invisible" part of the modular boundary consisting of the $\Gamma$-orbits of irrational points in $\mathbf{P}^{1}(\mathbf{R})$. The space $B_{\Gamma}:=\Gamma \backslash \mathbf{P}^{1}(\mathbf{R})$ is an archetypal bad quotient which should be treated as a non-commutative space: see CoMar1, MaMar, Ma4, and references therein. Below we will discuss what objects should be considered as modular forms on $B_{\Gamma}$.

We will restrict ourselves by the basic case $\Gamma=G L(2, \mathbf{Z})$. There is another description of $B_{\Gamma}$ : it is the set of the equivalence classes of $\mathbf{R}$ modulo the equivalence relation

$$
x \equiv y \Leftrightarrow \exists m, n \text { such that } T^{m} x=T^{n} y
$$

where the shift operator $\mathrm{T}$ is defined by

$$
T: x \mapsto \frac{1}{x}-\left[\frac{1}{x}\right] .
$$

Consider instead the dual shift on functions given by the formal operator $L=L_{1}$ :

$$
(L h)(x):=\sum_{k=1}^{\infty} \frac{1}{(x+k)^{2}} f\left(\frac{1}{x+k}\right) .
$$

The meaning of dualization in this context is clarified by the following formula:

$$
\int_{[0,1]} f \cdot L h d x=\int_{[0,1]}(f \mid T) h d x .
$$

Generalizing (3.3), we can introduce the formal operator $L_{s}$ on functions $\mathbf{R} \rightarrow \mathbf{C}$ :

$$
\left(L_{s} h\right)(x):=\sum_{k=1}^{\infty} \frac{1}{(x+k)^{2 s}} h\left(\frac{1}{x+k}\right) .
$$

To put it more conveniently, consider

$$
L:=\sum_{k=1}^{\infty}\left[\begin{array}{ll}
0 & 1 \\
1 & k
\end{array}\right] \in \mathbf{Z}\left[G L(2, \mathbf{Z}) \varsigma^{ץ}\right.
$$

where the hat means an obvious localization of the group ring. This operator acts on the space of $s$-densities $h(d x)^{s}$. Then:

$$
L\left(h(d x)^{s}\right)=L_{s} h(d x)^{s}
$$


and moreover

$$
\int_{[0,1]} f(d x)^{1-s} \cdot L\left(h(d x)^{s}\right)=\int_{[0,1]}\left(f(d x)^{1-s} \mid T\right) h(d x)^{s} .
$$

We now take as our heuristic principle the following prescription:

an $L$-invariant $s$-density is a substitute of a modular form of weight $2-2 s$ on the non-commutative modular curve $B$.

Notice that our motivation for adopting this principle consists of two steps: first, we replace the action of $\Gamma$ by that of $T$ (in view of (3.2)); second, we dualize. Roughly speaking, we replace invariant vectors by invariant functionals.

The classical example is Gauss 1-density

$$
\frac{1}{\log 2} \frac{1}{1+x}
$$

which appeared in Gauss' famous conjecture on the distribution of continued fractions.

In the following we will briefly describe recent work of D. Mayer (|May $)$, J. Lewis, and D. Zagier ([LZ1, LZ2]), giving a very beautiful description of the spectrum of values of $s$ for which an $L$-invariant density exists. The construction starts with D. Mayer's discovery of a space on which $L_{s}$ becomes an honest trace class operator.

3.4. Mayer's operators. Mayer's space V is defined as the space of holomorphic functions in $\mathbf{D}=\left\{z \in \mathbf{C}|| z-1 \mid<\frac{3}{2}\right\}$ continuous at the boundary. With the supremum norm, it becomes a complex Banach space.

3.4.1. Claim. (i) The formal operator $L_{s}$ for $\operatorname{Re} s>1 / 2$ is of trace class (in fact, nuclear of order 0) on the Banach space $\mathbf{V}$.

(ii) It has a meromorphic continuation to the whole complex plane of $s$, holomorphic except for simple poles at $2 s=1,0,-1, \ldots$.

(iii) The Fredholm determinant $\operatorname{det}\left(1-L_{s}^{2}\right)$ can be identified with the Selberg zeta function of $\operatorname{PSL}(2, \mathbf{Z}) \backslash H$.

For a proof, see May.

3.4.2. Corollary. L-invariant/antiinvariant $s$-densities which can be obtained by restriction from a density in $\mathbf{V}|d z|^{s}$ exist if and only if $s$ is a zero of the Selberg's zeta $Z(s)$.

All zeroes of $Z(s)$ can be subdivided into the following groups:

(i) $s=1$.

(ii) Zeroes on $\operatorname{Re} s=1 / 2$.

(iii) Zeroes $s=1-k, k=2,3,4, \ldots$.

(iv) Critical zeroes of Riemann's $\zeta(s)$ divided by two (hence on $\operatorname{Re} s=1 / 4$, if one believes the Riemann Hypothesis).

3.4.3. Theorem (Lewis-Zagier). (i) $s=1$ corresponds to the Gauss density $(1+x)^{-1}$.

(ii) Zeroes on $\operatorname{Re} s=1 / 2$ produce all real analytic $L^{2}$-invariant $s$-densities on $(0, \infty)$ tending to zero as $x \rightarrow+\infty$. They are automatically holomorphic on $\mathbf{C}-$ $(-\infty, 0])$.

(iii) Zeroes $s=1-k, k=2,3,4, \ldots$ produce all polynomial $L^{2}$-invariant densities, which are period functions of modular forms of integral weight on the upper half-plane. 
(iv) The s-densities corresponding to the critical zeroes of Riemann's zeta are analytic continuations of "half Eisenstein series"

$$
h_{s}(z)=\sum_{m, n \geq 1}(m(z+1)+n)^{-2 s} .
$$

A moral of this beautiful story from our perspective is this: the non-trivial zeroes of $Z(s)$ furnish a spectrum of complex fractal dimensions; are there spaces behind them?

\section{$\S 4$. Fractional dimensions in homological algebra}

4.1. Introduction. A "dimension", or "weight", in homological algebra is simply a super/subscript of the relevant (co)homology group. Cohomology groups are invariants of a complex, considered as an object of a derived/triangulated category. Terms of a complex are routinely graded by integers (at least, up to a shift), and the differential is of degree \pm 1 . Are there situations where we get a fractional numbering?

The answer is positive. Such situations arise in the following way.

A derived category $D(\mathcal{C})$ of an abelian category $\mathcal{C}$ may have other abelian subcategories $\mathcal{C}^{\prime}$ satisfying certain compatibility conditions with the triangulated structure and called "hearts" of the respective $t$-structures (see $[\mathrm{BeBD}]$ ).

For any heart $\mathcal{C}^{\prime}$, there is a natural cohomology functor $H^{\mathcal{C}^{\prime}}: D(\mathcal{C}) \rightarrow \mathcal{C}^{\prime}$.

Objects of such hearts can be considered as "perverse modifications" of the initial objects of $C$ represented by certain complexes of objects of $C$. In this way, perverse sheaves were initially defined via perversity functions by R. MacPherson. He has also invented a construction which translates the algebraic notion of perversity function on triangulated spaces into a geometric notion of perverse triangulation and revives Euclid's original intuition in the context of refined perversity: cf. $\mathrm{Vy}$ ]. In this context the dimension remains integral.

However, some very common derived categories $D(\mathcal{C})$, for example coherent sheaves on an elliptic curve, have families of hearts $\mathcal{C}^{\theta}$ indexed by real numbers $\theta$.

This "flow of charges" was first discovered in the context of Mirror Symmetry (cf. [Dou]). For a mathematical treatment due to T. Bridgeland, see [Br]; one can also find there some physics comments.

The values of the respective homology functor $H^{\theta}: D(\mathcal{C}) \rightarrow \mathcal{C}^{\theta}$ then naturally can be thought as having "dimension/weight $\theta$ ".

Below I will describe a particular situation where such groups appear in the framework of non-commutative tori and Real Multiplication program of [Ma5] developed in $[\mathrm{Po} 2$.

4.2. CR-lattices. In the Real Multiplication program, I suggested consideration of pseudolattices which are groups $\mathbf{Z}^{2}$ embedded in $\mathbf{R}$ as "period lattices of noncommutative tori", in the same way as discrete subgroups $\mathbf{Z}^{2} \subset \mathbf{C}$ are period lattices of elliptic curves. Here I will start with introducing the category of $\mathbf{C R}$-lattices combining properties of lattices and pseudolattices.

Objects of this category are maps $(j: P \rightarrow V, s)$, embeddings of $P \cong \mathbf{Z}^{3}$ into 1-dim $\mathbf{C}$-space $V$, such that the closure of $j(P)$ is an infinite union of translations of a real line; $s$ denotes a choice of its orientation.

(Weak) morphisms are commutative diagrams 


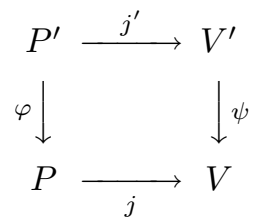

where $\psi$ is a linear map. Strong morphisms should conserve the orientations.

Each CR-lattice is isomorphic to one of the form

$$
P_{\theta, \tau}: \mathbf{Z} \oplus \mathbf{Z} \theta \oplus \mathbf{Z} \tau \subset \mathbf{C}, \theta \in \mathbf{R}, \operatorname{Im} \tau \neq 0 .
$$

We have $P_{\theta, \tau} \cong P_{\theta^{\prime}, \tau^{\prime}} \Longleftrightarrow \theta^{\prime}=\frac{a \theta+b}{c \theta+d}$ for some $\left(\begin{array}{ll}a & b \\ c & d\end{array}\right) \in G L(2, \mathbf{Z})$, and $\tau^{\prime}=$ $\frac{\tau+e \theta+f}{c \theta+d}$ for some $e, f \in \mathbf{Z}$.

We will discuss the following problem:

Define and study non-commutative spaces representing quotient spaces $\mathbf{C} /(\mathbf{Z} \oplus$ $\mathbf{Z} \theta \oplus \mathbf{Z} \tau)$.

4.2.1. Approach I: via non-commutative tori. (i) Produce the quotient $\mathbf{C} /(\mathbf{Z} \oplus$ $\mathbf{Z} \theta$ ) interpreting it as a $C^{\infty}$ non-commutative space, quantum torus $T_{\theta}$, with the function ring $A_{\theta}$ generated by the unitaries

$$
U_{1}, U_{2}, U_{1} U_{2}=e^{2 \pi i \theta} U_{2} U_{1} .
$$

(ii) Introduce "the complex structure" on $A_{\theta}$ as a non-commutative $\bar{\partial}$-operator $\delta_{\tau}:$

$$
\delta_{\tau} U_{1}:=2 \pi i \tau U_{1}, \delta_{\tau} U_{2}:=2 \pi i U_{2} .
$$

Denote the resulting space $T_{\theta, \tau}$.

4.2.2. Approach II: via elliptic curves. (i) Produce the quotient $\mathbf{C} /(\mathbf{Z} \oplus \mathbf{Z} \tau)$ interpreting it as the elliptic curve $S_{\tau}$, endowed with a real point

$$
x_{\theta}:=\theta \bmod (\mathbf{Z} \oplus \mathbf{Z} \tau) .
$$

(ii) Form a "non-commutative quotient space" $S_{\tau, \theta}:=S_{\tau} /\left(x_{\theta}\right)$ interpreting it via a crossed product construction in the language of non-commutative spectra of twisted coordinate rings, or $q$-deformations of elliptic functions, etc.

4.2.3. Problem. In what sense do the two constructions produce one and the same non-commutative space $T_{\theta, \tau} "=" S_{\tau} /\left(x_{\theta}\right)$ ?

This particular situation is an example of a deeper problem, which more or less explicitly arises at all turns in non-commutative geometry: at our disposal there is no good, or even working, definition of morphisms of non-commutative spaces. Worse, we do not quite know what are isomorphisms between non-commutative spaces. Even more concretely, assume that our non-commutative space is a quotient of some common space $M$ with respect to an action of two commuting groups, say, $G$ and $H$, such that $G \backslash M$ exists as an honest commutative space upon which $H$ acts in a "bad" way, and similarly $M / H$ exists as an honest commutative space upon which $G$ acts in a "bad" way. We can interpret the non-commutative space $G \backslash M / H$ via a crossed product construction applied either to $(G \backslash M) / H$ or to $G \backslash(M / H)$. However, these two constructions generally produce quite different non-commutative rings. 
According to the general philosophy, these two rings then are expected to be Morita equivalent in an appropriate sense, that is, have equivalent categories of representations. For an example where such a statement is a theorem, see Rieffel's paper $[\mathrm{Rie}$.

Polishchuk's answer to problem 4.2.3 is in this spirit, but more sophisticated. We will succinctly state it right away and then produce some explanations in subsections 4.3-4.6:

4.2.4. Claim (A. Polishchuk). $T_{\theta, \tau}$ and $S_{\tau} /\left(x_{\theta}\right)$ have canonically equivalent categories of coherent sheaves which are defined as follows:

On $T_{\theta, \tau}$ : the category of vector bundles, that is projective modules over $T_{\theta}$ endowed with a $\delta_{\tau}$-compatible complex structure.

On $S_{\tau, \theta}$ : the heart $\mathcal{C}^{-\theta^{-1}}$ of the $t$-structure of the derived category of coherent sheaves on the elliptic curve $\mathbf{C} /(\mathbf{Z}+\mathbf{Z} \tau)$ associated with the slope $-\theta^{-1}$.

4.3. Holomorphic structures on modules and bimodules. Let $A_{\theta}$ be the function ring of a $C^{\infty}$ non-commutative torus (4.1), endowed with a complex structure (4.2). A right $A_{\theta}$-module $E$ can be geometrically interpreted as vector bundle on this torus.

A holomorphic structure, compatible with $\delta_{\tau}$, on a right $A_{\theta}$-module $E$ is defined as a map $\bar{\nabla}: E \rightarrow E$ satisfying

$$
\bar{\nabla}(e a)=\bar{\nabla}(e) a+e \delta_{\tau}(a)
$$

Holomorphic maps between modules are maps commuting with $\bar{\nabla}$. Similar definitions can be stated for left modules (see [Co1, PoS, Po1, Po2]).

We imagine modules endowed with such a holomorphic structure as (right) vector bundles on the respective holomorphic torus. Their cohomology groups can be defined à la Dolbeault: $H^{0}(E):=\operatorname{Ker} \bar{\nabla}, H^{1}(E):=\operatorname{Coker} \bar{\nabla}$.

Similarly, a projective $A_{\theta}-A_{\theta^{\prime}}$-bimodule $E$ can be imagined as a sheaf on the product of two smooth tori. If both tori are endowed with holomorphic structures, and $E$ is endowed with an operator $\bar{\nabla}$ compatible with both of them, this sheaf descends to the respective holomorphic non-commutative space. It can be considered now as a Morita morphism $T_{\theta, \tau} \rightarrow T_{\theta^{\prime}, \tau^{\prime}}$, that is, the functor $E \otimes *$ from the category of right vector bundles on $T_{\theta, \tau}$ to the one on $T_{\theta^{\prime}, \tau^{\prime}}$. This agrees very well with motivic philosophy in commutative algebraic geometry, where morphisms between, say, complete smooth varieties are correspondences.

For two-dimensional non-commutative tori, there are good classification results for these objects.

4.3.1. $C^{\infty}$-classification of projective $A_{\theta}$-modules. Fix $(n, m) \in \mathbf{Z}^{2}$. Define the right $A_{\theta}$-module $E_{n, m}(\theta)$ as $A_{\theta}^{|n|}$ for $m=0$ and as the Schwartz space $S(\mathbf{R} \times \mathbf{Z} / m \mathbf{Z})$ with right action

$$
f U_{1}(x, \alpha):=f\left(x-\frac{n+m \theta}{m}, \alpha-1\right), f U_{2}(x, \alpha)=\exp \left(2 \pi i\left(x-\frac{\alpha n}{m}\right)\right) f(x, \alpha),
$$

for $m \neq 0$. These modules are projective.

4.3.2. Claim. Any finitely generated projective right $A_{\theta}$-module is isomorphic to $E_{n, m}(\theta)$ with $n+m \theta \neq 0$. 
We define the degree, rank, and slope of $E_{n, m}(\theta)$ by the formulas

$$
\operatorname{deg} E_{n, m}(\theta):=m, \operatorname{rk} E_{n, m}(\theta):=n+m \theta, \mu\left(E_{n, m}(\theta)\right):=\frac{m}{n+m \theta} .
$$

Notice that rank is generally fractional: according to the von Neumann - Murray philosophy, it is the normalized trace of a projection.

$E_{n, m}(\theta)$ is isomorphic to $E_{-n,-m}(\theta)$. It is sometimes convenient to introduce a $\mathbf{Z}_{2}$-grading declaring $E_{n, m}(\theta)$ even for $\operatorname{deg} E_{n, m}(\theta)>0$ and odd for $<0$.

Basic modules are defined as $E_{n, m}(\theta)$ for $(n, m)=1$. Generally, $E_{n d, m d}(\theta) \cong$ $E_{n, m}(\theta)^{d}$.

It is convenient to do bookkeeping using matrices instead of vectors. For $g \in$ $S L(2, \mathbf{Z})$, write $E_{g}(\theta):=E_{d, c}(\theta)$ where $(c, d)$ is the lower row of $g$. Accordingly, set

$$
\operatorname{deg} g:=c, \operatorname{rk}(g, \theta):=c \theta+d,
$$

and we have

$$
\operatorname{rk}\left(g_{1} g_{2}, \theta\right)=\operatorname{rk}\left(g_{1}, g_{2} \theta\right) \operatorname{rk}\left(g_{2}, \theta\right), g \theta:=\frac{a \theta+b}{c \theta+d} .
$$

4.3.3. Claim. The endomorphisms of a basic module $E_{g}(\theta)$ form an algebra isomorphic to $A_{g \theta}$ :

$$
V_{1} f(x, \alpha)=f\left(x-\frac{1}{c}, \alpha-a\right), V_{2} f(x, \alpha)=\exp \left(2 \pi i\left(\frac{x}{c \theta+d}-\frac{\alpha}{c}\right)\right) f(x, \alpha) .
$$

Thus $E_{g}(\theta)$ is a biprojective $A_{g \theta}-A_{\theta}$ bimodule. Tensor multiplication by it produces a Morita isomorphism of the respective quantum tori.

4.4. Holomorphic structures. Now endow $A_{\theta}$ with the holomorphic structure (4.2). There is a one-parametric series of compatible holomorphic structures on $E_{n, m}(\theta), m \neq 0$. Namely, let $z \in \mathbf{C}$. For $f$ in the Schwartz space put

$$
\bar{\nabla}_{z}(f):=\partial_{x} f+2 \pi i(\tau \mu(E) x+z) f .
$$

Similarly, for $E=A_{\theta}$ as right module put

$$
\bar{\nabla}_{z}(a):=2 \pi i z a+\delta_{\tau}(a) .
$$

In fact, $\bar{\nabla}_{z}$ up to isomorphism depends only on $z \bmod (\mathbf{Z}+\mathbf{Z} \tau) / \mathrm{rk} E$.

The Leibniz rule for the left action of $A_{g \theta}$ reads

$$
\bar{\nabla}_{z}(b e)=b \bar{\nabla}_{z}(e)+\frac{1}{\operatorname{rk} E} \delta_{\tau}(b) e .
$$

4.4.1. Theorem. Right holomorphic bundles on $T_{\theta, \tau}$ with arbitrary complex structures compatible with $\delta_{\tau}$ form an abelian category $\mathcal{C}_{\theta, \tau}$.

Every object of this category admits a finite filtration whose quotients are isomorphic to standard bundles (basic modules with a standard structure).

Let us now turn to elliptic curves. We start with some cohomological preliminaries. 
4.5. Torsion pairs. Let $\mathcal{C}$ be an abelian category.

$A$ torsion pair in $\mathcal{C}$ is a pair of full subcategories $p=\left(\mathcal{C}_{1}, \mathcal{C}_{2}\right)$ stable under extensions, with $\operatorname{Hom}\left(\mathcal{C}_{1}, \mathcal{C}_{2}\right)=0$ and such that every $A \in \mathcal{C}$ has a unique subobject in $\mathcal{C}_{1}$ with quotient in $\mathcal{C}_{2}$.

(ii) The t-structure on $D(\mathcal{C})$ associated with this torsion pair is defined by

$$
\begin{aligned}
D^{p, \leq 0} & :=\left\{K \in D(\mathcal{C}) \mid H^{>0}(K)=0, H^{0}(K) \in \mathcal{C}_{1}\right\}, \\
D^{p, \geq 1} & :=\left\{K \in D(\mathcal{C}) \mid H^{<0}(K)=0, H^{0}(K) \in \mathcal{C}_{2}\right\} .
\end{aligned}
$$

(iii) The heart of this $t$-structure is a new abelian category $\mathcal{C}^{p}:=D^{p, \leq 0} \cap D^{p, \geq 0}$. It is endowed with a torsion pair as well, namely $\left(\mathcal{C}_{2}[1], \mathcal{C}_{1}\right)$ (the tilting).

Now let $X$ be a smooth complete algebraic curve. Slope of a stable bundle on $\mathrm{X}$ is defined as deg/rk, slope of a torsion sheaf is $+\infty$. The category $\mathrm{Coh}_{I}$, by definition, consists of extensions of sheaves with slope in $I \subset \mathbf{R}$.

For any irrational $\theta$, the pair $\left(\mathrm{Coh}_{>\theta}, \mathrm{Coh}_{<\theta}\right)$ is a torsion pair in the category of coherent sheaves on a curve $X$ ( $\theta$ irrational).

Denote by $\operatorname{Coh}^{\theta}(X)$ the heart of the respective $t$-structure.

4.5.1. Theorem. The category $\mathcal{C}_{\theta, \tau}$ defined in Theorem 4.4 .1 is equivalent to $\mathrm{Coh}^{-\theta^{-1}}\left(T_{0, \tau}\right)$.

4.6. Real multiplication case. In the real multiplication case, $\theta$ is a real quadratic irrationality. The crucial observation characterising such $\theta$ is this:

$\theta$ is a real quadratic irrationality $\Longleftrightarrow$

$\exists g \in S L(2, \mathbf{Z})$ such that $g \theta=\theta \Longleftrightarrow$

$E_{g}(\theta)$ is a biprojective $A_{\theta}-A_{\theta}$ bimodule, inducing a non-trivial autoequivalence of the category of $A_{\theta}$-modules.

Polishchuk remarked that such a bimodule, endowed with a holomorphic structure, determines a non-commutative graded ring

$$
B:=\oplus_{n \geq 0} H^{0}\left(E_{g}(\theta)_{A_{\theta}}^{\otimes n}\right) .
$$

This ring is a particular case of a more general categorical construction. Let $\mathcal{C}$ be an additive category, $F: \mathcal{C} \rightarrow \mathcal{C}$ an additive functor, $O$ an object.

Then we have a graded ring with twisted multiplication:

$$
A_{F, O}:=\oplus_{n \geq 0} \operatorname{Hom}_{\mathcal{C}}\left(O, F^{n}(O)\right) .
$$

To produce the former $B$, choose $O=A_{\theta}, F(\cdot)=\cdot \otimes E_{g}(\theta)$ in the category of holomorphic bundles.

4.6.1. Theorem (A. Polishchuk). For every real quadratic irrationality $\theta$ and complex structure $\tau$, the heart $\operatorname{Coh}^{\theta}\left(T_{0, \tau}\right)$ is equivalent to the Serre category of right coherent sheaves on the non-commutative projective spectrum of some algebra of the form $A_{F, O}$ with $F: D^{b}\left(T_{0, \tau}\right) \rightarrow D^{b}\left(T_{0, \tau}\right)$ being an autoequivalence.

This remarkable result may be tentatively considered as an approximation to the problem invoked in Ma5]: how to find finitely generated (over $\mathbf{C}$ and eventually over $\mathbf{Z}$ ) rings naturally associated with $T_{\theta, \tau}$. They are necessary to do arithmetics of quantum tori with real multiplication along the lines of the classical complex multiplication theory.

Since however an arbitary parameter $\tau$ appears in this construction, the following question remains: how to choose $\tau$ for arithmetical applications?

A natural suggestion is: if $\theta \in \mathbf{Q}(\sqrt{d})$, choose $\tau \in \mathbf{Q}(\sqrt{-d})$. 
For such a choice, Polishchuk's non-commutative rings can be considered as a more sophisticated version of Kronecker's idea to merge $\sqrt{d}$ with $\sqrt{-d}$ in order to produce solutions of Pell's equation in terms of elliptic functions: cf. A. Weil We for a modern exposition and historical context.

\section{ABout THE AUTHOR}

Yuri Manin is a professor at Northwestern University and a member of the board of directors of the MPI at Bonn. He is a winner of the Nemmers Prize and a member of the American Academy of Arts and Sciences.

\section{REFERENCES}

[And] G. Anderson. Cyclotomy and a covering of the Taniyama group. Comp. Math., 57 (1985), 153-217. MR0827351 (88e:11045)

[At] M. Atiyah. Commentary on the article of Yu. I. Manin: "New dimensions in geometry". In: Springer Lecture Notes in Math., 1111, 1985, 103-109. MR0797417 (87j:14031)

[BeBD] A. Beilinson, J. Bernstein, P. Deligne. Faisceaux pervers. Astérisque, 100 (1982), 5171. MR0751966 (86g:32015)

[Br] T. Bridgeland. Stability conditions on triangulated categories. Preprint math.AG/0212237

[Co1] A. Connes. $C^{*}$ algèbres et géométrie differentielle. C. R. Acad. Sci. Paris, Sér. A-B, 290 (1980), A599-A604. MR0572645 (81c:46053)

[Co2] A. Connes. Noncommutative Geometry. Academic Press, 1994. MR1303779 (95j:46063)

[Co3] A. Connes. Geometry from the spectral point of view. Lett. Math. Phys. Vol. 34 (1995), 203-238. MR 1345552 (96j:46074)

[Co4] A. Connes. Gravity coupled with matter and the foundation of non-commutative geometry. Comm. Math. Phys., 182 (1996), no. 1, 155-176. MR1441908 (98f:58024)

[Co5] A. Connes. Trace formula in noncommutative geometry and the zeros of the Riemann zeta function. Selecta Math. (N.S.), 5 (1999), no. 1, 29-106. MR.1694895|(2000i:11133)

[Co6] A. Connes. Cyclic cohomology, quantum group symmetries and the local index formula for $S U_{q}(2)$, J. Inst. Math. Jussieu, 3 (2004), no. 1, 17-68. MR2036597 (2005f:58044)

$[\mathrm{CoKr}] \quad$ A. Connes, D. Kreimer. Renormalization in quantum field theory and the RiemannHilbert problem. I. The Hopf algebra structure of graphs and the main theorem. Comm. Math. Phys., 210:1 (2000), 249-273. MR1748177 (2002f:81070)

[CoMar1] A. Connes, M. Marcolli. Quantum statistical mechanics of Q-lattices. (From Physics to Number Theory via Noncommutative Geometry, Part I). Preprint math.NT/0404128

[CoMar2] A. Connes, M. Marcolli. Renormalization and motivic Galois theory. Int. Math. Res. Notices, No. 76 (2004), 4073-4092. MR 2109986

[CoMos] A. Connes, H. Moscovici. The local index formula in noncommutative geometry. GAFA Vol. 5 (1995), No. 2, 174-243. MR.1334867 (96e:58149)

[ConsMar] C. Consani, M. Marcolli. Noncommutative geometry, dynamics, and $\infty$-adic Arakelov geometry. Selecta Math. (N.S.), 10 (2004), no. 2, 167-251. MR.2080121 (2005g:58055)

[DaLSSV] L. Dạbrowski, G. Landi, A. Sitarz, W. van Suijlekom, J.C. Varilly. The Dirac operator on $S U_{q}(2)$. Preprint math.QA/0411609.

[De1] C. Deninger. Local L-factors of motives and regularized determinants. Inv. Math., 107 (1992), 135-150. MR1135468 (93a:11056)

[De2] C. Deninger. Motivic L-functions and regularized determinants. Proc. Symp. Pure Math., 55:1 (1994), 707-743. MR.1265547 (94m:11077)

[De3] C. Deninger. Some analogies between number theory and dynamical systems on foliated spaces. Doc. Math. J. DMV. Extra volume ICM I (1998), 23-46. MR1648030 (99g:11084)

[De4] C. Deninger. A note on arithmetic topology and dynamical systems. Contemp. Math. 300, AMS, Providence, RI (2002), 99-114. MR.1936368 (2004c:14030) 
[Dou] M. Douglas. Dirichlet branes, homological mirror symmetry, and stability. In: Proceedings of the ICM 2002, Higher Education Press, Beijing, 2002, vol. III, 395-408. Preprint math.AG/0207021. MR/1957548(2004c:81200)

[Ha] M. Haran. The mysteries of the real prime. Clarendon Press, Oxford, 2001. MR:1872029 (2003b:11085)

[He] T. L. Heath. The thirteen books of Euclid's Elements. Translation, Introduction and Commentary. Cambridge UP, 1908.

[KalW] W. Kalau, M. Walse. Gravity, non-commutative geometry and the Wodzicki residue, J. Geom. Phys., 16 (1995), 327-344. MR1336738 (96c:58016)

[KapSm] M. Kapranov, A. Smirnov. Cohomology determinants and reciprocity laws: number field case. Unpublished.

[Ka] N. Katz. p-adic properties of modular schemes and modular forms. Springer LNM, 350 (1973). MR0447119 (56:5434)

[Kr] D. Kreimer. On the Hopf algebra structure of perturbative Quantum Field Theory. Adv. Theor. Math. Phys., 2:2 (1998), 303-334. MR1633004 (99e:81156)

[LaPo] M. Lapidus, C. Pomerance. Fonction zêta de Riemann et conjecture de Weyl-Berry pour les tambours fractals. C. R. Acad. Sci. Paris, Sér. I Math., 310 (1990), 343-348. MR 1046509 (91d:58248)

[LavF1] M. Lapidus, M. van Frankenhuysen. Complex dimensions of fractal strings and oscillatory phenomena in fractal geometry and arithmetic. In: Spectral Problems in Geometry and Arithmetic (ed. by T. Branson), Contemp. Math., vol. 237, AMS, Providence, RI, 1999, 87-105. MR1710790 (2001c:11093)

[LavF2] M. Lapidus, M. van Frankenhuysen. Fractal Geometry and number theory. Complex dimensions of fractal strings and zeros of zeta functions. Birkhäuser, 2000. MR1726744 (2001b:11079)

[LZ1] J. Lewis, D. Zagier. Period functions for Maass wave forms. Ann. of Math., 153 (2001), 191-258. MR.1826413 (2003d:11068)

[LZ2] J. Lewis, D. Zagier. Period functions and the Selberg zeta function for the modular group. In: The mathematical beauty of physics, Adv. Series in Math. Physics, 24, World Sci. Publ., River Edge, NJ (1997), 83-97. MR1490850 (99c:11108)

[Mand] B. Mandelbrot. The fractal geometry of nature. Freeman \& Co., NY, 1982. MR0665254 (84h:00021)

[Ma] D. Yu. Manin. Personal communication

[Ma1] Yu. Manin. New dimensions in geometry. Russian: Uspekhi Mat. Nauk, 39:6 (1984), 47-73. English: Russian Math. Surveys, 39:6 (1984), 51-83; and Springer Lecture Notes in Math., 1111, 1985, 59-101. MR0771098|(86d:14002) MR0797416 (87j:14030)

[Ma2] Yu. Manin. Three-dimensional hyperbolic geometry as $\infty$-adic Arakelov geometry. Inv. Math., 104 (1991), 223-244. MR1098608 (92f:14019)

[Ma3] Yu. Manin. Lectures on zeta functions and motives (according to Deninger and Kurokawa). In: Columbia University Number Theory Seminar, Astérisque, 228 (1995), 121-164. MR:1330931 (96d:11076)

[Ma4] Yu. Manin. Von Zahlen und Figuren. Preprint math.AG/0201005.

[Ma5] Yu. Manin. Real multiplication and noncommutative geometry. In: The legacy of Niels Henrik Abel, ed. by O. A. Laudal and R. Piene, Springer Verlag, Berlin, 2004, 685-727. Preprint math.AG/0202109. MR2077591

[MaMar] Yu. Manin, M. Marcolli. Continued fractions, modular symbols, and non-commutative geometry. Selecta Math., new ser., 8 (2002), 475-521. Preprint math.NT/0102006. MR:1931172 (2004a:11039)

[May] D. Mayer. Continued fractions and related transformations. In: Ergodic Theory, Symbolic Dynamics and Hyperbolic Spaces, Eds. T. Bedford et al., Oxford University Press, Oxford, 1991, 175-222. MR1130177

[Maz] B. Mazur. Note on étale cohomology of number fields. Ann. Sci. ENS, 6 (1973), 521552. MR0344254 (49:8993)

[Mor] M. Morishita. On certain analogies between knots and primes. Jour. für die reine u. angew. Math., 550 (2002), 141-167. MR1925911 (2003k:57008)

[PoS] A. Polishchuk, A. Schwarz. Categories of holomorphic bundles on noncommutative two-tori. Comm. Math. Phys., 236 (2003), 135-159. Preprint math.QA/0211262. MR:1977884 (2004k:58011) 
[Po1] A. Polishchuk. Noncommutative two-tori with real multiplication as noncommutative projective varieties. Preprint math.AG/0212306.

[Po2] A. Polishchuk. Classification of holomorphic vector bundles on noncommutative twotori. Preprint math.QA/0308136.

[Rie] M. Rieffel. Von Neumann algebras associated with pairs of lattices in Lie groups. Math. Ann., 257 (1981), 403-418. MR0639575 (84f:22010)

[Se] J.-P. Serre. Formes modulaires et fonctions zêta p-adiques. Springer LNM, 350 (1973), 191-268. MR0404145 (53:7949a)

[Sm1] A. Smirnov. Hurwitz inequalities for number fields. St. Petersburg Math. J., 4 (1993), 357-375. MR.1182400 (93h:11065)

[Sm2] A. Smirnov. Letters to Yu. Manin of Sept. 29 and Nov. 29, 2003.

[Sou] C. Soulé. Les variétés sur le corps à un elément. Moscow Math. Jour., 4:1 (2004), 217-244. MR:2074990 (2005h:14002)

[Ta] J. Tate. Duality theorems in Galois cohomology over number fields. Proc. ICM, Stockholm, 1962, 288-295. MR0175892 (31:168)

[Ti] J. Tits. Sur les analogues algébriques des groupes semi-simples complexes. Colloque d'Algèbre sup., Bruxelles 1956; Louvain, 1957, 261-289. MR0108765 (21:7477)

[Vy] M. Vybornov. Constructible sheaves on simplicial complexes and Koszul duality. Math. Res. Letters, 5 (1998), 675-683. MR.1666864 (2000h:18021)

[We] A. Weil. Elliptic functions according to Eisenstein and Kronecker. Springer Verlag, Berlin, 1976 and 1999. MR0562289 (58:27769a)

Northwestern University, Evanston, Illinois, USA

Current address: Max-Planck-Institut für Mathematik, Vivatsgasse 7, 53111 Bonn, Germany 